\title{
CONCEPTS FOR SCADA SYSTEMS PLANNING IN A CHANGING COMPETITIVE ENVIRONMENT
}

\author{
Jorge M. SANTOS, Aníbal T. de ALMEIDA \\ EDP Distribuição, Energia, S.A., ISR-Universidade de Coimbra - Portugal \\ jorge.mendessantos@edis.edp.pt, adealmeida@isr.uc.pt
}

\section{INTRODUCTION}

The electric sector liberalization is creating structural and technological changes in the utilities, pushing the classical SCADA models and other utility systems to an evolving process. There are new needs to be provided, namely data exchange between companies and between different sectors of the same company, remote maintenance, remote equipment configuration, telemetering, Demand Side Management, Distributed Generation, House/Building Automation, etc., which will create new challenges to utility SCADA and communication engineers.

A proposed architecture solution is presented which is able to provide the required flexibility to cope with all the above mentioned requirements in a cost-effective way.

\section{PRESENT AND FUTURE SCADA PROTOCOLS}

SCADA systems and protocols were developed from multiple manufacturers and vendors, all of them with the same purpose but with different technologies. This diversity represents a big problem to utilities, namely to upgrade and to achieve a proper interconnection. All of the available systems were proprietary and difficult/costly to match. In the 90's, a new project was sponsored by EPRI to promote interoperability between utilities computer systems, taking advantage of computer and network developments. The project team was constituted by an expert group with a broad industry and utility involvement and was known by the UCA project (Utility Communications Architecture) [1]. Its main purpose was specifying a suite of internationally recognized open communication protocols that met the utility industry communication requirements, including electric, gas and water utilities, and use off-the-shelf international standards to reduce costs and raise interoperability. UCA presented a new perspective of SCADA protocols to the utility community because it differs from most previous utility protocols in its use of object models of devices and device components, and standard communication protocols. The models define common data formats, identifiers and controls for substation and feeder devices. The UCA architecture allows for multivendor interoperability, easiness of integration, reduced integration costs, more efficient use of bandwidth, use of the OSI model with an open architecture, real-time operation, etc. The UCA work resulted in several concepts standardized by IEC and adopted by the main equipment manufacturers, and gradually applied in utilities.

The SCADA planning job is now simplified at the technology choice, being the best selection to use international IEC standards. These standards are supported by the well known IP protocol and Internet Technology (IT) devices. For example, IEC 60870-6 (also known as ICCP or TASE.2) standard is used for SCADA systems interconnection, and IEC 61968 standard used for data exchange between utility systems like SCADA, maintenance, planning, meter reading and control, network optimization, records and asset management systems. IEC 60870-6 standard is intended to support inter-application integration when there is a need to exchange data on an event driven basis. Also the intrasubstation communication was contemplated with standardized protocols. The IEC 61850 protocol was created to avoid the inter-manufacturer device communication problem and to perform all the communication necessities between Intelligent Electronic Devices (IEDs) inside substations. This standard is based also in the IP protocol and Ethernet network, with several topology possibilities. Even the IEC60870-5-101, the first SCADA standardized protocol for substation interconnection, initially conceived to low bandwidth channels, was upgraded to IEC60870-5-104 for IP networks.

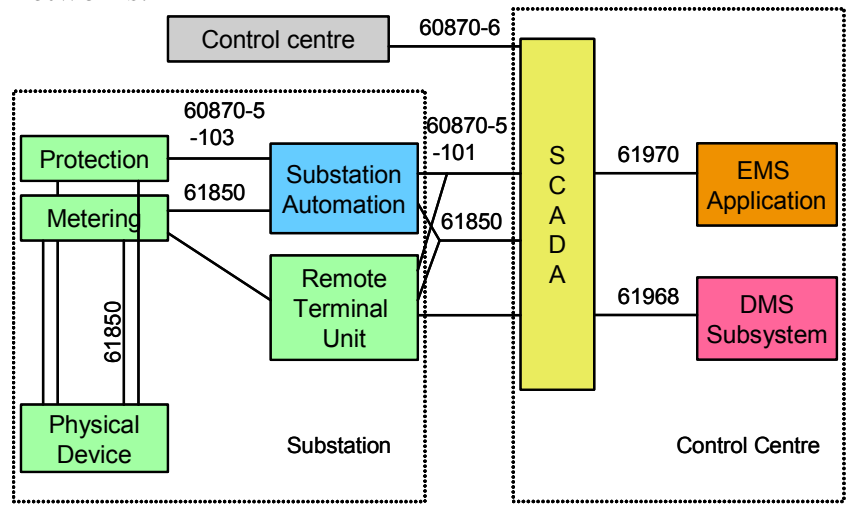

Figure 1 - Utility IEC standard protocols for Control Center and Substation [2]

Since the UCA project start in the early 90's, several aspects changed in the SCADA world. While in the past SCADA engineers were concentrated in picking-up the best system among a wide number of choices, comparing functionalities, characteristics, interfaces, components, etc., nowadays the main objective is to guarantee interoperability, making the best system the most open one which may not always be the most complete at the initial stage. There is definitively an important turnover in SCADA networks. However, the standard is not sufficiently restrictive for blind choice. It foresees a group of selectable parameters to tune the protocol with the application characteristic, which must be performed by SCADA engineers.

The main change and challenge to SCADA engineers is to plan IP networks to substitute old and recent SCADA networks, based on analog or serial digital channels. These networks have some characteristic antagonistic with IP networks. For example, no latency, no need for address planning, easy interconnection due to few parameters configuration, static routes. These were constituted by multiple point-to-point channels that perform a fixed bus topology, generally without automatic reconfiguration or 
assured by high hierarchy equipments like SDH (Synchronous Digital Hierarchy).

In addition to standard SCADA protocols, remote maintenance and equipment setting, file assessment and transfer and other services like communication VoIP channels (Voice over IP), sharing the same IP platform are contributing to labour efficiency and to cost reductions. Almost all new remote devices, applied in substations and in feeder infrastructures, are microprocessor-based devices, and some of them allow data recording and posterior remote data access (oscilography, event registration, and diverse measure data). For this to be true there is a need for a well planned IP network, with adequate bandwidth, good redundancy, high reliability, with low latency and high security. To make possible future network enlargements, increase personal mobility and permit future functionalities, a Network instead of several networks has to be created. This network has to support all services needed at the remote infrastructure, including FTP (File Transfer Protocol), SCADA data exchange in real-time, remote maintenance and equipment setting services, remote video and building surveillance, telephony services (VoIP), remote access to stored data. Eventually, videoconferencing with technical personal for remote support can be used, in a concept similar to telemedicine or space-shuttle remote assistance system.

Depending on services supported, especially in substationlike LAN (Local Area Network), it can be necessary to use dedicated networks for some highly mission-critical relay communications to assure the Quality of Service (QoS) necessary to trip commands. This specific network can have backup assured by the other relay interface connection used for the remote maintenance and FTP services. The WAN (Wide Area Network) network topology should be meshed, to create alternative ways to data flow.

\section{UTILITY TYPICAL COMMUNICATIONS}

Typically utilities use several communication media, namely, twisted pair cable, fiber optic cable (OPGW ${ }^{1}, \mathrm{ADSS}^{2}$, wrapped types, micro-cable), DLC (Distribution Line Carrier) and PLC (Power Line Carrier), public operator leased line, analog and digital radio networks, trunked mobile radio networks, switched public operator networks, either fixed or cellular [3].

Each one of these technologies has advantages and disadvantages, being used according to the technical-economic analysis made for each case. The analog communication channel can just be used by non-IP services, such as voice and low bandwidth data connections. Some digital communication technologies became very popular in recent years, because they support all communication services that utilities need. It is possible to simultaneously have both IP technologies and analog or digital low bandwidth channels, in the same system. For example, fiber optic cables and digital transmission technologies (SDH and PDH Plesiochronous Digital Hierarchy) are being widely used for backbone utility communication links and main places interconnection, due to its versatility and wide bandwidth characteristic. These technologies support a great diversity of interfaces, like, analog and digital channels, voice and data, all within the same platform. New WDM (Wavelength Division Multiplexing) fiber optic technologies are being developed, which can be used for backbone communication

1 Optical Power Ground Wire

2 All Dielectric Self Supporting bandwidth upgrade. All of these technologies support IP encapsulation either directly or indirectly, and have bandwidth from hundred of Mbps to the hundred of Gbps range.

Radio is another very popular medium used in utilities for voice and data communications. The most widely used bands are VHF and UHF with diverse topologies, from point-to-point links to point-to-multipoint networks. The first topology is used for cheap remote site coverage, with low bandwidth requirements. The point-to-multipoint solution is very used for wide area coverage, with several sites geographically dispersed or mobile voice communications.

\section{NEW CHALLENGES FOR THE UTILITIES}

In the recent past and in the near future electrical utilities have to address new market and operational challenges, which will need to be supported by SCADA systems and the associated data networks. The liberalization process creates more data interconnection needs. It will be necessary to exchange data between utilities, some of them geographically overlapped, to measure the energy provided to customers who can switch between service providers more dynamically. Implementation of concepts, such as Distributed Generation (DG), Demand Response (DR) and Demand Side Management (DSM) programs, will change the electrical distribution networks dispatch, creating more data exchange necessities. The implementation and the continued work of Distribution Automation (DA) will also contribute to an exponential growth of the number of points to be controlled. DA is of fundamental importance to improve power quality, which would benefit from gathering more information, at a higher rate, and by having more automatic processes to minimize the interruption times. The DA sites can inform about the fault locations to help dispatch personnel to isolate electrical faults and to allow field personnel to arrive faster to the place of the fault. If the communication is fast enough, and there is information from the nearby switches, the fault location can be isolated automatically by a local control system, avoiding human intervention.

The DG and the DA have in common the geographical dispersion characteristic. [4] It is expected that in the future DG will transform the distribution networks, with energy sources such as wind, solar, natural gas CHP having a large impact on the energy supply by 2010 . The liberalized market creates more metering necessities for billing purposes. Depending on the tariff choice and the market rules, it can be necessary to acquire daily or hourly electricity measurements, which make impracticable manual or switched telephone line metering methods. The sites to be measured have some requisite similarity with DR, DSM and DA, on bandwidth and communication link characteristic.

The DR and DSM programs need telemeter systems to be efficient. The real-time and bidirectional communication characteristic are the keystone for the success of these programs. DR and DSM can be applied to medium and large consumers or can be extended to residential and commercial consumers. The main difference between them is that, for medium and large consumers, large energy savings can be made with few consumers (and few communication links), while for residential and commercial consumers, it will be necessary a great number of communication links to achieve similar energy savings.

The inclusion of Demand Response programs in energy markets can take the form of several mechanisms, such as reduced energy costs, direct payments for energy not 
consumed, and reservation payment for being available to reduce consumption upon request. Also the time periods can vary from a continuous base time (Real Time Prices) to peak and off-peak time periods [5].

Previous studies conclude that the end-users involvement is very important to the success of DR programs [6]. The best way to involve the residential and commercial end-users is recurring to Home Automation (HA) or Building Automation (BA) systems. The load control in accordance with the signals received from the utility is carried out by the HA/BA system, without interfering with the customer comfort. HA/BA is penetrating the market, with several systems available. [7]. In Europe, the KNX standard (Konnex), supported by KNX association which is resulting of the merge of three associations (BCI - BatiBUS Club International, EIBA European Installation Bus Association, and EHSA - European Home Systems Association) [8], is the leading technology and has already a wide acceptance in the market. It has also a large industry support by the main European electrical device manufacturers. Other technologies are being developed all over the world with the same purpose, such as, bacnet [9], lonworks [10] and X10 [11]. This means that, in a near future, residential and commercial buildings will change from passive electricity consumers to become intelligent or active electric systems. This is an opportunity to DR and DSM programs application. A main requirement is a communication interface between utility and the building intelligent systems, including the correspondent data protocol and services. The electrical devices can be separated in two groups - controllable outputs and non- controllable outputs. The last group includes all outputs (electrical devices such as non-intelligent household appliances, fundamental illumination, etc.) that can not be controlled or that is essential. The other group includes intelligent household appliances, secondary illumination, HVAC systems, etc. To accomplish these concepts, consumers have to participate actively in DR and DSM programs, allowing utilities to control some authorized loads and appliances in their houses, fulfilling an initial agreement between the consumer and utility. The innovation here is that the consumer can dynamically customize its electrical installation with the desired timing, setting the comfort level (for example, heating temperature and rooms, dispensable illumination, etc.) and loads to contribute to the energy reduction. On the other way, intelligent household appliances can have an automatic function that allows to be controlled directly by the utility data signals, without sacrificing the comfort and security levels. For example, the refrigerator can have a temperature hysteresis that can inhibit the motor start in a "red" period. However, if the temperature falls bellow the minimum level, the motor starts even it has the "red" utility signal.

The house-utility interface can also include the energy meter function and can provide the utility the outage information as well as the instantaneous estimate of the dispensable energy, allowing the central utility DSM system to estimate all the available energy that can be used in the dispatching process. The installation can be optimized to lower the consumption, to flatten the consumption, to avoid peaks or to move the consumption peaks into cheap energy time intervals.

The house automation intelligent systems, receives the DR/DSM signals with the required power levels. For example, the utility can send a numerical value corresponding to the power to be reduced in each house instead of sending a "red" code that would globally reduce an unknown amount of energy.

In spite of HA/BA not having in the present a strong relationship with SCADA systems, it can be in the future a fundamental piece in the implementation of DR and DSM programs.

The traditional utility communication architectures will be insufficient to attend all data exchange and all site interconnection. From a communication point of view, it is necessary to find private, public, or both, communication solutions to accomplish the new requirements. On the other way, from the utility and SCADA side, it is necessary to have standardized interconnected platforms to merge all of this data and protocols in only one universe, allowing all data acquired (SCADA, telemetering, DA, etc.) to be directly available to all systems that need them. The system architecture required to integrate all these equipment and data coordination processes requires a substantial change in the SCADA networks.

There are other improvements that can be made to minimize the energy interruption periods and to optimize the utilities profits. The field teams should be provided with advanced communication facilities and portable devices, in such a way that they can have a mobile office with them. To accomplish that, it will be necessary to have a laptop, a printer, a handheld PDA, a GPS device, a mobile radio and a wireless data communication access to utility data network. With these equipments, the field personal can receive geographical digital maps, work plan information, electrical actualized grid schemes from the main database, work instructions, SCADA filtered information in real time (for example to search faults). After the intervention, they can upgrade the field information on-line in the utility network database.

Additionally, knowing the fault location, a maintenance support system can check in the database what components are necessary to repair the fault. With the handheld device, the technicians should maintain actualized, in the vehicle database, all the components used. This database would exchange data with the main maintenance system to actualize the asset management system. The GPS device would be useful for dispatch vehicle location, which permits to call the closest team, and on the other way, it can be interconnected with the PDA navigator software which chooses the best route to the fault place, allowing to reduce the response time.

\section{SCADA SYSTEM AND NETWORK PLANNING}

There are several emergent changes in the utility systems and services, which have to be accompanied by SCADA and communication utility systems planning [12].

From SCADA point of view, there is a substantial work already done by expert groups and standardized by IEC. The main standards for SCADA systems application are:

- IEC 60870-6, also known by TASE.2 or ICCP, used for Inter-Center communications;

- IEC 60870-5-101, already wide used for substation control center interconnection with low bandwidth channels;

- IEC 60870-5-103, used for protection interconnection;

- IEC 60870-5-104, the youngest of this series, used for substation control center interconnection through IP channels; - IEC 61850, developed for intra-substation device communication, can also be used in the future for control center substation interconnection, if there is not bandwidth limitations;

- IEC 61970, normalizes a set of Application Program Interfaces (API) for the manipulation of real-time critical, near real-time and historical EMS/SCADA data;

- IEC 61968, define interfaces to major elements of an interface architecture for Distributed Management Systems (DSM) 
- IEC/TR 62195, is a technical report about power system control and associated communications intended for deregulated energy market communications;

- IEC/TR 62210, is another technical report about power system control and associated communications, focused in data and communication security aspects for protocols used within and between utility systems;

These standards and technical reports were issued by the Technical Committee 57 [13], which is the committee working on power systems management and associated information exchange. Thus, for the conventional utility SCADA systems, the way to follow is the adoption of international standards for accomplishing multi-vendor and multi-system interoperability.

\section{Proposed SCADA/DSM/DR development}

For the new programs, such as DSM and DR, it is necessary to standardize data objects, models and protocols which permit to achieve a global system interconnection, multi-vendor independent and easily expandable. The architecture should be distributed, with local DSM concentrators which receive commands from the SCADA system and forward them to the HA/BA management systems in the downlink direction. For uplink, the DSM concentrators receive measure data from $\mathrm{HA} / \mathrm{BA}$, process it and send it to the SCADA system. The DSM concentrators can be located at substation level, depending on the number of customers in the DSM program and the used communication medium. With a distributed architecture, it is possible to extend the DSM program to a wide number of consumers, without overloading the SCADA processors and interfaces with a large amount of data. The data exchange between SCADA system and DSM concentrators relative to DSM program should be minimal. In the upload direction the DSM data can be calculated for different substation switchgear and power bars. For the download direction just one setpoint command to one of these points would be necessary. With this setpoint information, the DSM concentrator can manage the necessary commands to be sent to HA/BA systems, in order to achieve the global value sent by the SCADA system.

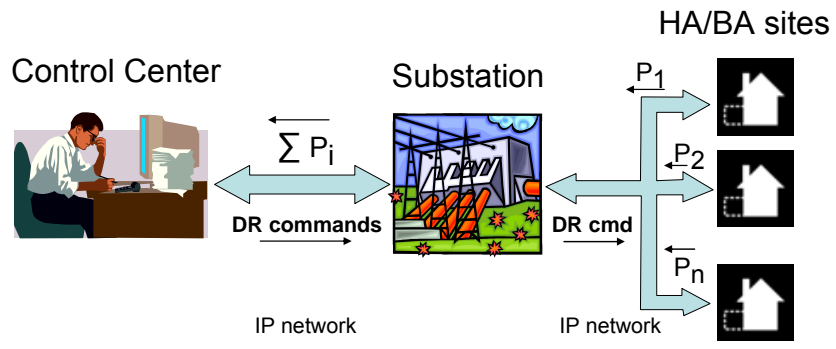

Figure 2 - SCADA/DSM/DR proposed architecture

\section{Communication Planning}

The utility communication networks have to be redesigned to support all of these systems. The main infrastructures have to be connected by wide bandwidth channels (SDH, PDH or WDM), forming the utility backbone communication structure.

For secondary sites, or where it is not necessary large bandwidth channels, digital alternative technologies, such as PLC, private trunked digital networks (TETRA in Europe) and cellular $3 \mathrm{G}$ public mobile networks can be considered. These last two technologies are very promising for future application in utility systems. PLC is still an expensive technology but it does not require a dedicated cabling. It is perfect for low signal wireless zones. In Europe, TETRA networks are being developed and improved to support voice and IP data connections, and can be of fundamental importance to new protocol adoption and to new service implementation, especially for DA and DG sites.

As the equipment and location number rises, it may be difficult to have a private communication system economically and technically viable for all locations and bandwidth requirements. Public telecom operators offer large bandwidth channels almost all over the country at low cost and with acceptable reliability. Its use for secondary location connection and for backup purposes to support important sites can be interesting. However, the security aspects become more critical when supported by public networks because of Internet promiscuous environment. It can be an issue that public operators have to guarantee in order to provide adequate security to the utilities.

The DR and DSM implementation policy depends very much on the number of adherent sites. To achieve an expressive effect of these programs, it is necessary to have a permanent communication medium that permits constant bidirectional data exchange between the utility and the clients, using a low cost communication solution. This can be achieved by large bandwidth connections and communicative meters (with Bluetooth or another wireless or easily connectable technology) or with new services provided by utilities offering Internet access, like Broadband Power Line (BPL). BPL is based on a technology (PLC) well known by utilities, but for low bandwidth and long distance links. However, the characteristic needed for DR and DSM is the opposite, i.e. short distance and high bandwidth. BPL is the ideal technology for DR and DSM services together with Internet service provider, because utilities have already the network infrastructure that can be economically more profitable. There are several ongoing experiences for BPL technologies all over the world [14], but this technology is not yet mature. There are several problems to solve before BPL can assume itself as a broadband Internet access and utilities as Internet service providers. Until then, mobile cellular public networks (3G) or broadband Internet customer access can be used for data transfer. For device interconnection inside the building, a wireless technology, such as Bluetooth can be used.

\section{CONCLUSION}

The competitiveness requirements of liberalization demands that utilities be more efficient, obliging them to simplify and improve many procedures and systems. The international standard protocol adoption is very important to achieve cost and time reductions in SCADA systems implementation and maintenance. Good network planning is also a fundamental issue, allowing easy system interconnection, network upgrades and expansions.

Distributed Generation, Demand Side Management, Demand Response and Distribution Automation expansion will oblige to new communication solutions, with large bandwidth and high number of sites requirements. Public and private communication channels can be used.

The House/Building Automation technologies can contribute to an inexpensive large scale implementation of DR and DSM programs, recurring eventually to Broadband Power Line or public communication networks to Internet services and DR/DSM data transfer. 
The standardization effort in SCADA systems will definitely contribute to utility success in achieving those goals. Telecommunication and computer networks will be of main importance for the necessary structural changes.

\section{REFERENCES}

\section{[1] http://www.ucausersgroup.org}

[2] Tom Berry, "Bi-Directional End Point Information Systems”, www.telmark.org/2002Sep/3-4_Berry.ppt

[3] Donald J. Marihart, 2001, “Communications Technology Guidelines for EMS/SCADA Systems”, IEEE Transactions on Power Delivery, Vol. 16, No.2, April 2001.

[4] Johan Enslin, 2004, “Interconnection of Distributed Power to the Distribution Network", Proceedings Power System Conference, Transmission and Distribution Poster Session A.

[5] Rosse Malme, 2004, "IEA Demand Response Resources project”, Proceedings Power System Conference, Panel 8.

[6] Sunil Cherian and Ron Ambrosio, 2004, "Towards Realizing the GridWise ${ }^{\mathrm{TM}}$ Vision: Integrating the Operations and Behavior of Dispersed Energy Devices, Consumers, and Markets”, Proceedings Power System Conference, Emerging Technologies in Power Engineering Poster Session A.

[7] Adrian Dusa, Geert Deconinck and Ronnie Belmans, 2004, "Communication System for Intelligent Residential Electrical Installations”, Proceedings Power System Conference, Power System Communications Poster Session A.
[8] http://www.konnex.org

[9] http://www.bacnet.org

[10] http://www.echelon.com

\section{[11] http://www.x10.com}

[12] T. Kostic, C. Frei, O. Preiss, M. Kezunovic, 2004, "Scenarios for Data Exchange using Standards IEC 61970 and IEC 61850", UCA User Group meeting, Cigre Paris Session 2004.

\section{[13] http://www.iec.ch}

[14] Weilin Liu, Hanspeter Widmer, Philippe Raffin, 2003, "Broadband PLC Access Systems and Field Deployment in European Power Line Networks”, IEEE Communication Magazine, 114 - 118. 